\title{
Decision support system for the glaucoma using Gabor transformation
}

\author{
U. Rajendra Acharya ${ }^{\mathrm{a}, \mathrm{b}}$, E.Y.K. $\mathrm{Ng}^{\mathrm{c}}$, Lim Wei Jie Eugene ${ }^{\mathrm{a}}$, Kevin P. Noronha ${ }^{\mathrm{d}, *}$, \\ Lim Choo Min ${ }^{\mathrm{a}}$, K. Prabhakar Nayak ${ }^{\mathrm{d}}$, Sulatha V. Bhandary ${ }^{\mathrm{e}}$
}

a Department of Electronics and Computer Engineering, Ngee Ann Polytechnic, Singapore

${ }^{\mathrm{b}}$ Department of Biomedical Engineering, Faculty of Engineering, University of Malaya, Malaysia

c School of Mechanical and Aerospace Engineering, Nanyang Technological University, Singapore

${ }^{\mathrm{d}}$ Department of E\&C, MIT Manipal, India

e Department of Ophthalmology, KMC, Manipal, India

A R T I C L E I N F O

Article history:

Received 10 June 2014

Received in revised form 28 August 2014

Accepted 16 September 2014

Available online 1 October 2014

\section{A B S T R A C T}

Increase in intraocular pressure (IOP) is one of the causes of glaucoma which can lead to blindness if not detected and treated at an early stage. Glaucoma symptoms are not always obvious; hence patients seek treatment only when the condition progressed significantly. Early detection and treatment will decrease the chances of vision loss due to glaucoma. This paper proposes a novel automated glaucoma diagnosis method using various features extracted from Gabor transform applied on digital fundus images. In this work, we have used 510 images to classify into normal and glaucoma classes. Various features namely mean, variance, skewness, kurtosis, energy, and Shannon, Rényi, and Kapoor entropies are extracted from the Gabor transform coefficients. These extracted features are subjected to principal component analysis (PCA) to reduce the dimensionality of the features. Then these features are ranked using various ranking methods namely: Bhattacharyya space algorithm, $t$-test, Wilcoxon test, Receiver Operating Curve (ROC), and entropy. In this work, $t$-test ranking method yielded the highest performance with an average accuracy of $93.10 \%$, sensitivity of $89.75 \%$ and specificity of $96.20 \%$ using 23 features with Support Vector Machine (SVM) classifier. Also, we have proposed a Glaucoma Risk Index (GRI) developed using principal components to classify the two classes using just one number.

(c) 2014 Elsevier Ltd. All rights reserved.

\section{Introduction}

Glaucoma damages the optic nerve of the eye because of the high intraocular pressure produced due to the imbalance in the production and drainage of the aqueous humor of the eye. The condition progresses with time, and will eventually lead to permanent blindness, if left untreated [1]. There is no cure for glaucoma, but medication can be used to prevent vision loss [2]. Normally, aqueous humor flows out of the eye via the trabecular meshwork. However, when this channel is blocked, the aqueous humor wil build up. The rise in this fluid will cause the pressure to build up and results in the death of ganglion cells [3]. Since the ganglion cells are not regenerated, glaucoma patients slowly lose their vision. Study has shown that there will be more than $30 \%$ increase in glaucoma cases by 2020 [4]. Currently, it is the second leading cause of blindness worldwide. The World Health Organization lists

\footnotetext{
* Corresponding author. +919845538161

E-mail addresses: kevinkurkal@yahoo.co.in, kevinkurkal@gmail.com (K.P. Noronha).

http://dx.doi.org/10.1016/j.bspc.2014.09.004

1746-8094/@ 2014 Elsevier Ltd. All rights reserved.
}

glaucoma as one of the major causes of visual impairment globally [5].

Open-angle glaucoma and angle-closure glaucoma are the two main types of glaucoma [6]. The open-angle glaucoma is the most common form of glaucoma. Since it is painless and progress at a slower rate, patients might only notice the loss of vision after the condition progressed significantly. In contrast, the angle-closure glaucoma is painful and sudden, and vision is lost at a significant rate [7]. However, due to the noticeable symptoms, patients will seek treatment before permanent damage occurs.

Glaucoma diagnosis in the early stage is very crucial in saving vision of the patient. Hence a routine screening of patients might help in detecting the disease in the early stage. Glaucoma is diagnosed by measuring IOP, visual fields and optic nerve head (ONH). Goldmann applanation tonometer can be used to measure IOP [8], Humphrey field analyzer can be used to analyze the visual fields [9] and fundus images to evaluate the cup to disk ratio of the $\mathrm{ONH}$ [10]. Measuring IOP and visual fields requires specialized instruments and hence is not suited for mass screening programs where large numbers of patients are required to be screened [11]. ONH appearance can be an ideal method to detect glaucoma which can 
be used in the mass screening. Ophthalmoscopy or stereo fundus photography of $\mathrm{ONH}$ can easily detect these changes and are widely used by ophthalmologists to document the disk, rim, cup areas and disk diameter [12].

In recent years digital fundus photography has played a major role in screening the diabetic retinopathy patients [13-16]. The digital fundus photography is non-invasive, cheap and can be used to diagnose different eye abnormalities like glaucoma, diabetes retinopathy, maculopathy and age related macular degeneration. Hence they are ideal for mass screening of glaucoma [17]. This automated diagnosis can also reduce the inter-observer and intraobserver variability errors arising during the screening of the disease by clinicians. Various morphological features like disk, cup and rim areas, disk diameter and cup to disk (C/D) ratio can be easily extracted from the digital fundus image [18].

While screening the glaucoma using $\mathrm{ONH}$ appearance, cup to disk ratio is used as an important parameter [19] in detecting the glaucoma. A cup size of a healthy person is very small as it contains more than 1.2 million fibers passing through it. When a person is affected by glaucoma, the $\mathrm{ONH}$ loses its optic nerve fibers resulting in the increase of cup size. This in turn increases the cup to disk ratio, indicating a glaucomatous change [20]. The cup to disk ratio in a healthy person is usually 0.3 [21]. As the C/D ratio increases, the severity of the glaucoma also increases. A mild glaucoma can have a C/D ratio of 0.4 [22]. A moderate glaucoma can have a C/D ratio of $0.5-0.7$. A severe glaucoma can have a $C / D$ ratio of above 0.7 . The mild stage is characterized by normal visual field or very less vision loss (side or peripheral). In case of moderate glaucoma even though the C/D ratio is around $0.5-0.7$, the central vision may not be affected. The severe stage is characterized by a severe loss of optic nerve fiber, the presence of focal notches (inferior or superior) [23] and optic nerve hemorrhages. The patient may lose the vision if this is uncontrolled. Typical normal, mild, moderate and severe glaucomatous fundus images are as shown in Fig. 1.

In recent years computer aided diagnosis (CAD) is playing a major role in screening the glaucoma. The CAD system is simple, repetitive, not prone to inter or intra observer variability and fast in diagnosis [24]. Also CAD can screen many patients in a small time. There is a scarcity of ophthalmologists in many developing countries, where CAD can be very useful. The proposed decision support system for glaucoma can differentiate normal and glaucoma classes accurately. This does not depend on the segmentation of individual $\mathrm{ONH}$ features like cup, disk or abnormalities like focal notches, optic nerve hemorrhages where a small error in the segmentation may lead to wrong diagnosis [25]. This is a nonsegmentation technique which depends on only on the extracted features, number of images and design of classifiers.

In the literature to calculate the C/D ratio authors have segmented optic disk and cup. The optic disk detection are based on template matching [26], Fuzzy convergence [27], contour model based approaches [28], geometric parametric model [29], and Hough transform [30]. Joshi et al. [31] have used depth discontinuity model to detect the C/D ratio (CDR) with a mean CDR error of 0.09. Nayak et al. [32] have used morphological operations for the detection of C/D ratio. Xu et al. [33] have used sparsity based feature extraction technique and SVM classifier for the detection of $C / D$ ratio. Edge detection and thresholding technique are used for the detection of C/D ratio [34]. They have reported a mean CDR error of 0.14

Fig. 2 shows the block diagram of the proposed system which is divided into offline and online system. Image pre-processing is the first step in the offline mode where the entire sets of fundus images (normal and glaucoma) are pre-processed. Gabor transform is performed on the fundus images and a number of features such as moments, entropy and energy are extracted. To reduce the dimensionality of the extracted features principle component analysis is performed. Features are ranked using various ranking methods to select the minimum number of features to obtain the highest performance. The ground truth (given by the ophthalmologists) about the class to which the fundus image belongs as well as the extracted significant feature set are then fed to the classifier. The classifier performance is measured using a tenfold cross validation strategy. In the real time mode, the fundus images are pre-processed first and the significant features are extracted from them. The classifier classifies the fundus images into normal and glaucoma based on the extracted significant features.

The paper is organized as follows: Section 2 explains the Data acquisition of fundus images, Gabor transform, feature extraction, PCA and feature ranking and selection. SVM and NB classifiers and Glaucoma Risk Index are discussed in Section 3. The results obtained from the proposed technique are presented in Section 4. Section 5 discusses the results obtained and the paper concludes in the Section 6

\section{Methods}

\subsection{Data acquisition}

In this work, we have used 510 retinal images of normal (266), mild (72), moderate (86), and severe (86) glaucoma classes. These data were provided by the Kasturba Medical College, Manipal, India and taken using ZEISS retinal camera FF450plus. The computer interfaced to the camera converts these images to JPEC format with an image size of $2588 \times 1958$ pixels. The acquired fundus images are resized to $240 \times 180$ pixels, and are subjected to adaptive histogram equalization [35]. This is to ensure uniform contrast across the image for improving the classification results

\subsection{Gabor transform}

Gabor filtering involves convoluting Gabor kernel with image to reveal texture information of certain frequency and orientation [36]. Gabor kernels are multiplication of Gaussian and Fourier kernel [37]. Gabor kernels have properties that make it attractive for computer vision applications. These properties include its appealing simplicity, optimum joint spatial/spatial-frequency localization, and ability to simulate the behavior of two dimensional receptive fields of simple cells in the visual cortex (by isolating specific frequencies and orientations).

Let $h_{x y}$ be the value of the Gabor kernel at position $(x, y)$. Then $h_{x y}$ is defined by

$h_{x y}=A e^{-i(f x \cos \theta+f y \sin \theta)} \cdot e^{-0.5\left(\frac{R_{1}^{2}}{\sigma_{1}^{2}}+\frac{R_{2}^{2}}{\sigma_{2}^{2}}\right)}$

where $A$ is a constant, $f$ is the frequency, $\theta$ is the orientation, $R_{1}=x \cos \theta+y \sin \theta$, and $R_{2}=y \sin \theta-x \cos \theta$. Fig. 3 shows the real part of Gabor kernel with various orientations and frequencies.

In order to obtain more information about the image, multiple Gabor kernels are used to perform the convolution process to obtain coefficients with different frequencies and orientations information. Such collection of kernels is known as Gabor bank [38]. We follow the method proposed by Zheng et al. [39] for the selection of kernels to include in the Gabor bank. In our work, the Gabor bank of 21 images is created using two different frequencies and four different orientations $\left(0^{\circ}, 45^{\circ}, 90^{\circ}\right.$, and $\left.135^{\circ}\right)$

The two frequencies are given by [39]:

$f_{n}=\frac{(0.75 * p i)}{2^{(f-1)}} \quad$ where $n=1,2$

Hence we obtained $f_{1}=1.1781$ and $f_{2}=2.3562$. These 21 images are eight amplitude, eight phases, four maximum square sums and 


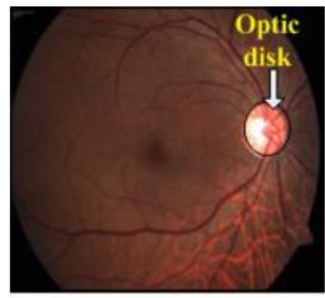

(a)

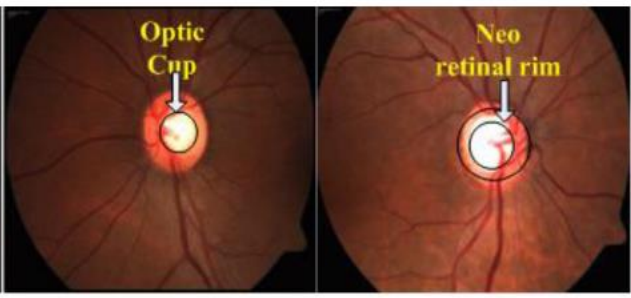

(b)

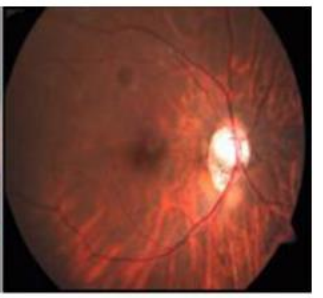

(d)

Fig. 1. Typical fundus images: (a) normal, (b) mild glaucoma, (c) moderate glaucoma, (d) severe glaucoma.

one image with index corresponding to maximum square sum. Four maximum square sums of images are generated from eight amplitudes corresponding to each orientation. Then one final image is created by assigning the index of image (among four images) to the maximum pixel among four images.

\subsection{Feature extraction}

Various features namely mean, variance, skewness, kurtosis, Shannon entropy, Rényi entropy, Kapoor entropy, and energy are extracted from the Gabor transform coefficients.
Statistical moments are used to describe the shape of distribution function [40]. In our work, the function is the probability density function of coefficients. The first four orders of statistical moments are mean $\mu$, variance $\sigma^{2}$, skewness $\gamma$, and kurtosis $\beta$. Suppose $P$ is the random 1 variable then the first four orders of statistical moments are computed by Eqs. (3)-(6).

$$
\mu=E[P]
$$

$$
\sigma^{2}=E\left[(P-\mu)^{2}\right]
$$

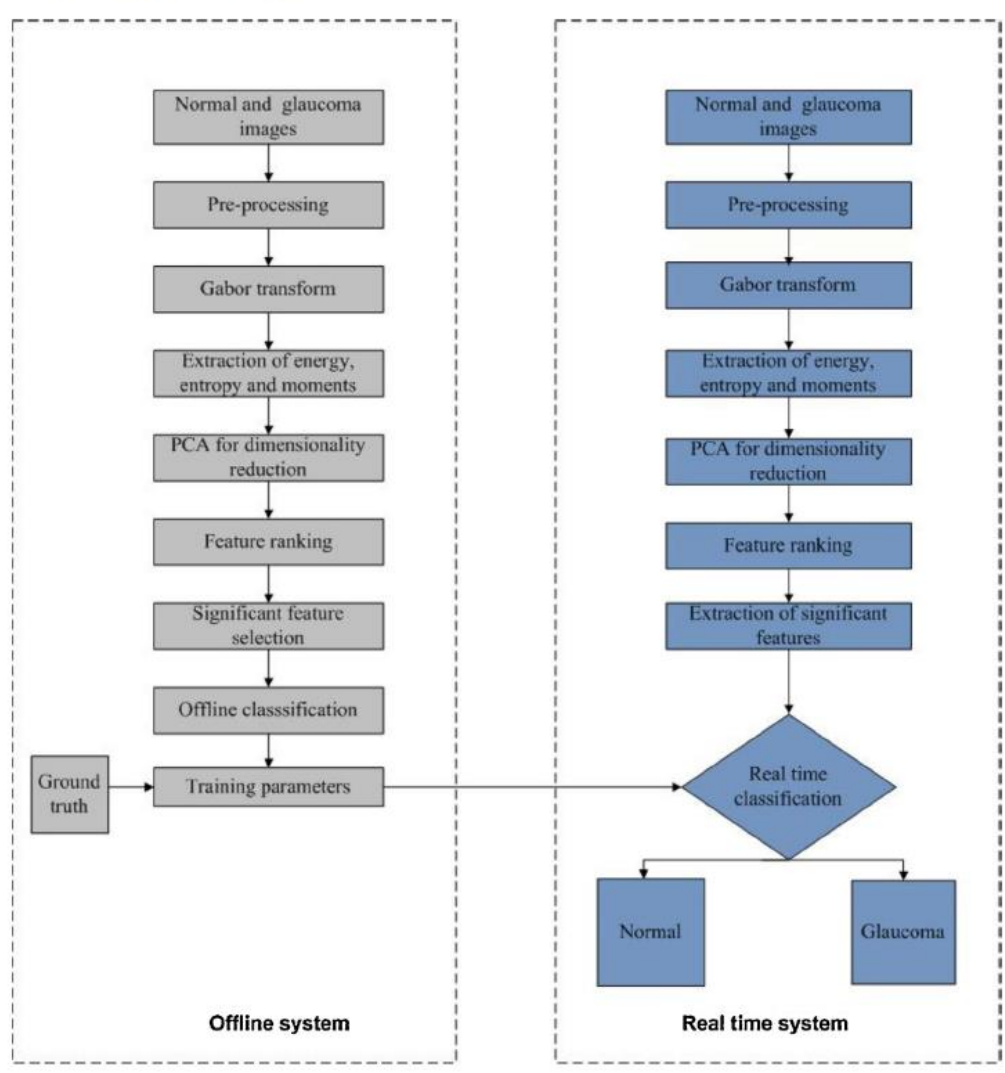

Fig. 2. The proposed system. 

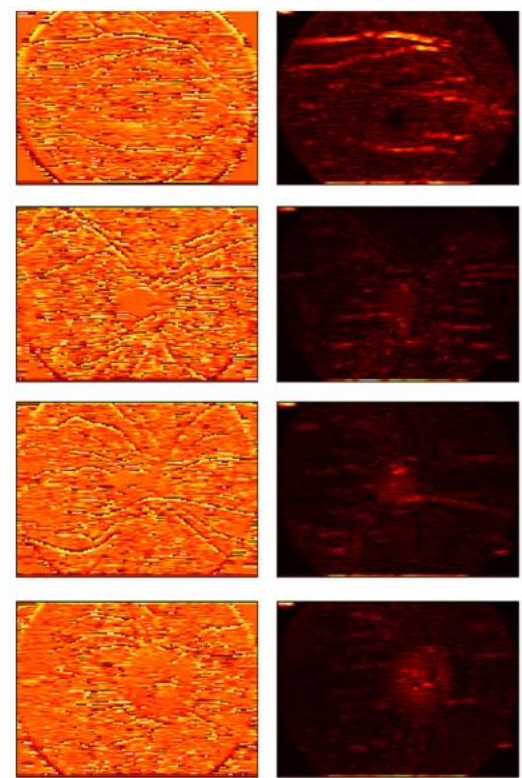
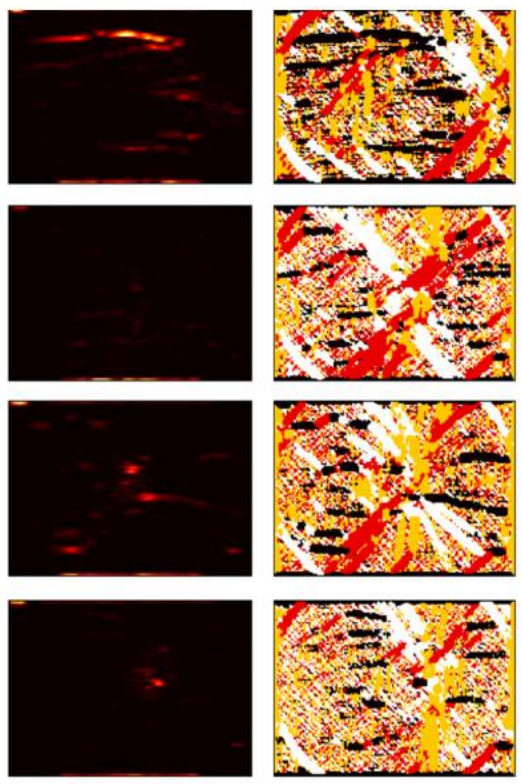

Fig. 3. Results of Gabor transform: (column 1) amplitude $\left(0^{\circ}\right)$, (column 2) phase $\left(0^{\circ}\right.$ ), (column 3 ) square sum, and (column 4 ) index corresponding to maximum square sum for (a) normal, (b) mild glaucoma, (c) moderate glaucoma and (d) severe glaucoma images.

$\gamma=E\left[\left(\frac{P-\mu}{\sigma}\right)^{3}\right]$

$\beta=\frac{E\left[(P-\mu)^{4}\right]}{\left(E\left[(P-\mu)^{2}\right]\right)^{2}}$

Entropy measures the amount of disorder of the coefficients, with zero representing equal distribution. Let $p$ is a vector such that $p_{i}$ represents probability of finding intensity values $i$ in signal $I$. The Shannon entropy $\left(H_{\mathrm{S}}\right)$, Rényi entropy $\left(H_{\mathrm{R}}\right)$ and Kapoor entropy $\left(H_{\mathrm{K}}\right)$ are computed using Eqs. (7), (8) and (9) respectively [41,42]. Note that $H_{\mathrm{R}}$ is parameterized by an order value $\alpha_{\mathrm{R}}$, and $H_{\mathrm{K}}$ is parameterized by $\alpha_{\mathrm{K}}$ and $\beta_{\mathrm{K}}$. In this work, we have used $\alpha_{\mathrm{R}}=3$, $\alpha_{\mathrm{K}}=0.5$ and $\beta_{\mathrm{K}}=0.7[41]$.

$H_{\mathrm{S}}=\sum_{i=1}^{N} p_{i} \log _{2} p_{i}$

$H_{\mathrm{R}}(\alpha)=\frac{1}{1-\alpha} \log _{2} \sum_{i=1}^{N} p_{i}^{\alpha}$

$H_{\mathrm{K}}(\alpha, \beta)=\frac{1}{\beta-\alpha} \log _{2} \frac{\sum_{i=1}^{N} p_{i}^{\alpha}}{\sum_{i=1}^{N} p_{i}^{\beta}}$

Energy is used to define the amount of information present in the image and is computed using Eq. (10).

Energy $=\sum_{i, j=1}^{N} p^{2}(i, j)$

\subsection{Principal component analysis (PCA)}

In PCA, a covariance analysis between factors is performed to reduce the data dimensionality. It is a transformation of large correlated variables into small number of uncorrelated variables called principal components (PC) [43]. The highest variability in the data can be obtained using the first PC and succeeding PC's account for remaining data variability. PCA computes the directions of highest variability and projects the data onto them. PCA starts with the computation of data covariance matrix, followed by the Eigen values and Eigen vectors computation and sorting the Eigen vectors in the descending order of Eigen value [44]. It concludes with actual data projections into the directions of sorted Eigen vectors.

In our work we have extracted a total 168 features after the feature extraction method. These 168 features are applied to PCA for dimensionality reduction yielding 32 features.

\subsection{Feature ranking and selection}

Feature ranking and selection is a critical step to select a subset of significant features from the entire set of features. We can select the minimum features to get the best classification accuracy if we know the ranking of the features. While retaining the features which have highest rank one can ignore the features with low rank and still can get highest accuracy. This also speeds up the classification process. In this work we have used $t$-test, Bhattacharyya space algorithm, Wilcoxon test, Receiver Operating Curve (ROC) and entropy ranking methods. The $t$-test method compares the population means of the two groups to identify the correlation among the features [45]. Chernoff bound provides exponential decay on tail distributions using independent variables. Hence it is used with Bhattacharyya distance for feature ranking [46]. The ROC based feature ranking selects the features using area between ROC and the random classifier slope. Wilcoxon method is 
Table

Results of principal components for normal and glaucoma classes ( $p$-value $<0.0001$ ).

\begin{tabular}{|c|c|c|c|c|}
\hline PCs & Normal & Glaucoma & $p$-value & $t$-value \\
\hline PC3 & $0.410 \pm 0.552$ & $0.442 \pm 0.655$ & $2.37 \mathrm{E}-46$ & 15.87 \\
\hline PC2 & $0.244 \pm 0.614$ & $0.263 \pm 0.879$ & $1.77 \mathrm{E}-13$ & 7.57 \\
\hline PC1 & $0.250 \pm 0.575$ & $0.270 \pm 1.212$ & $8.69 \mathrm{E}-10$ & 6.25 \\
\hline PC7 & $0.056 \pm 0.285$ & $0.060 \pm 0.329$ & $2.44 \mathrm{E}-05$ & 4.25 \\
\hline PC9 & $0.041 \pm 0.244$ & $0.044 \pm 0.262$ & 0.000146 & 3.82 \\
\hline PC10 & $0.031 \pm 0.234$ & $0.034 \pm 0.242$ & 0.001976 & 3.11 \\
\hline PC15 & $0.021 \pm 0.171$ & $0.023 \pm 0.188$ & 0.004707 & 2.83 \\
\hline PC22 & $0.014 \pm 0.137$ & $0.015 \pm 0.140$ & 0.01849 & 2.36 \\
\hline PC5 & $0.050 \pm 0.539$ & $0.054 \pm 0.487$ & 0.021897 & 2.29 \\
\hline PC20 & $0.014 \pm 0.142$ & $0.015 \pm 0.146$ & 0.023383 & 2.27 \\
\hline PC23 & $0.012 \pm 0.136$ & $0.013 \pm 0.133$ & 0.032688 & 2.14 \\
\hline PC28 & $0.009 \pm 0.106$ & $0.010 \pm 0.114$ & 0.03518 & 2.11 \\
\hline PC12 & $0.018 \pm 0.202$ & $0.020 \pm 0.230$ & 0.042176 & 2.03 \\
\hline PC29 & $0.008 \pm 0.104$ & $0.008 \pm 0.109$ & 0.076317 & 1.77 \\
\hline PC17 & $0.011 \pm 0.165$ & $0.012 \pm 0.159$ & 0.085501 & 1.72 \\
\hline PC25 & $0.009 \pm 0.114$ & $0.009 \pm 0.137$ & 0.086892 & 1.71 \\
\hline PC31 & $0.007 \pm 0.089$ & $0.007 \pm 0.108$ & 0.093996 & 1.67 \\
\hline PC26 & $0.006 \pm 0.120$ & $0.007 \pm 0.119$ & 0.17885 & 1.34 \\
\hline PC27 & $0.005 \pm 0.098$ & $0.005 \pm 0.131$ & 0.277392 & 1.08 \\
\hline PC18 & $0.006 \pm 0.143$ & $0.007 \pm 0.168$ & 0.29854 & 1.04 \\
\hline PC14 & $0.008 \pm 0.181$ & $0.008 \pm 0.195$ & 0.301992 & 1.03 \\
\hline PC16 & $0.007 \pm 0.184$ & $0.007 \pm 0.155$ & 0.313133 & 1.00 \\
\hline PC24 & $0.004 \pm 0.131$ & $0.004 \pm 0.133$ & 0.421662 & 0.80 \\
\hline PC6 & $0.011 \pm 0.306$ & $0.012 \pm 0.358$ & 0.428323 & 0.79 \\
\hline PC13 & $0.005 \pm 0.190$ & $0.005 \pm 0.208$ & 0.529399 & 0.62 \\
\hline PC4 & $0.011 \pm 0.476$ & $0.012 \pm 0.613$ & 0.621932 & 0.49 \\
\hline PC19 & $0.003 \pm 0.143$ & $0.003 \pm 0.156$ & 0.639847 & 0.46 \\
\hline PC11 & $0.003 \pm 0.225$ & $0.004 \pm 0.235$ & 0.706577 & 0.37 \\
\hline PC32 & $0.001 \pm 0.091$ & $0.001 \pm 0.0986$ & 0.767389 & 0.29 \\
\hline PC30 & $0.0006 \pm 0.092$ & $0.0007 \pm 0.110$ & 0.875614 & 0.15 \\
\hline PC21 & $0.0005 \pm 0.134$ & $0.0005 \pm 0.152$ & 0.934028 & 0.08 \\
\hline PC8 & $0.0006 \pm 0.223$ & $0.0007 \pm 0.299$ & 0.953301 & 0.05 \\
\hline
\end{tabular}

a non-parametric test, which does not assume normal distribution [47]. The basic idea of entropy method is to filter out those features whose expression distributions are relatively random. The features with smaller entropy are more discriminatory [48]. In this work, we have obtained the highest classification performance using 23 features with $t$-test ranking method out of the total 32 significant principal components.

\section{Classifiers}

In this work, we have used SVM and Naive Bayesian (NB) classifiers to select the best classifier for the automated diagnosis of glaucoma which are described below.

\subsection{SVM classifier}

SVM is a machine learning technique used for binary class supervised classification [49]. It attempts to fit a hyperplane through the feature space to discriminate between two classes linearly. In reality most of the data in the world are not separable linearly [50]. They can be best separable if we use the kernel functions. The kerne function can be used to map the feature points to higher dimension. In this work, we have used linear, polynomial, quadratic, and radial basis function kernel functions.

\subsection{Naive Bayesian Classifier}

Naïve Bayes classifier is a classifier that uses Bayes theorem [51] and supervised machine learning technique. Bayes theorem is used to compute the probability of certain event happening given certain confirmed condition [52]. The classification is based on the knowledge of the similar objects already classified known as training sets. The testing and training set objects are defined by their features.
3.3. Performance measures

The dataset used for this study is $\mathbf{5 1 0}$ fundus images (normal (266), mild (72), moderate (86), and severe (86) glaucoma classes). A tenfold cross validation strategy is employed to test the classifiers using the extracted features in view of the low sample size. The tenfold cross validation testing has a lower variance than a single fold testing. This is very important when we have a limited dataset. In a single hold out set, $90 \%$ of the data is used for training where as $10 \%$ of the data is used for testing. This makes the test sample size very small which might lead to a lot of variation in the performance estimation. A $k$-fold validation ( $k=10$ in our case) reduces this variance by averaging over $k$ different partitions, so the performance estimate is less sensitive to the partitioning of the data. In tenfold cross validation the entire datasets is divided into ten equal parts [53]. The process begins with the training of nine parts of the data and testing of the remaining one part. The procedure is repeated ten times testing different part each time. The accuracy, sensitivity and specificity are computed for each iteration. The average of all the tenfolds gives the actual average accuracy, sensitivity and specificity.

\subsection{Glaucoma Risk Index (GRI)}

From Table 1 it is observed that there is a uniform pattern between the selected features between normal and glaucoma classes and these selected features can be used to formulate a Glaucoma Risk Index (GRI). The range of index may provide the information about the classes. In the clinical context, a threshold can be set for these indexes to enable objective discrimination by the doctors as to whether the input retinal fundus image belongs to normal or glaucoma class. In this work, we have used a combination of ranked features (principal components) extracted from the fundus images to form the GRI. This index is computed using 
Table 2

Performance measures of all the classifiers using PCA components as input features.

\begin{tabular}{llllllll}
\hline Classifier & No. of features & TP & TN & FP & FN & Sn (\%) & Sp (\%) \\
\hline NB & 9 & 209 & 248 & 15 & 35 & 85.66 & 94.30 \\
SVM Poly 1 & 23 & 219 & 253 & 10 & 25 & 89.75 & 96.20 \\
SVM Poly 2 & 9 & 222 & 241 & 22 & 22 & 90.98 & 90.14 \\
SVM Poly 3 & 32 & 205 & 248 & 15 & 39 & 84.02 & 94.10 \\
SVM RBF & 9 & 222 & 232 & 31 & 22 & 90.98 & 88.30 \\
\hline
\end{tabular}

Notations used: TP, true positive; TN, true negative; FP, false positive, FN, false negative, Sn: densitivity; Sp: specificity; Acc: accuracy.

Eq. (11) such that the combination of the ranked features resulted in unique range for normal and glaucoma classes with maximum separation. We have obtained Eq. (11) by trial-and-error method. GRI is computed using the following formula:

$$
\begin{aligned}
\mathrm{GRI}= & 6.8375-1.1325(\mathrm{PC} 1)+1.6500(\mathrm{PC} 2)+2.7225(\mathrm{PC} 3) \\
& +0.6750(\mathrm{PC} 4)+0.6650(\mathrm{PC} 5)
\end{aligned}
$$

\section{Results}

The given set of fundus images were applied to a Gabor bank which consists of 2 different frequencies and 4 different orientations produces a total of 21 Gabor images. From these 21 images a set of 168 features $(21 \times 8$ features $)$ are extracted which include mean, variance, skewness, kurtosis, Shannon entropy, Rényi entropy, Kapoor entropy, and energy. The dimensionalities of the features are reduced to 32 by using PCA. Then these features are ranked using various ranking methods. In this work, we have obtained the highest classification performance using 23 features with $t$-test ranking method and SVM classifier. Table 1 shows the mean \pm standard deviation of the PCA features and Fig. 4 shows the plot of variances of PC's of normal and glaucoma classes. It can be seen from the Table 1 that, PC values are marginally higher for glaucoma classes compared to the normal classes.

The performance of the different ranking methods for different combination of features is shown in Fig. 5. In this work $t$-test yielded the highest performance using 23 features.

Fig. 6 shows performance measure using different classifiers for various folds of 10 -fold cross-validation. SVM classifier with polynomial 1 kernel yielded the highest classification accuracy of $93.10 \%$, sensitivity of $89.75 \%$ and a specificity of $96.20 \%$. Table 2
Table 3

Mean \pm SD of GRI values for normal and glaucoma subjects.

\begin{tabular}{llll}
\hline Feature & Normal & Glaucoma & $p$-value \\
\hline GRI & $8.68 \pm 1.67$ & $4.84 \pm 2.08$ & $<0.0001$
\end{tabular}

shows the classification results of the classifiers using features presented in Table 1.

Table 3 shows the GRI shows for normal and glaucoma classes and Fig. 7 shows the spread of index for two classes. The GRI for normal class is ( $8.68 \pm 1.67)$ and glaucoma is $(4.84 \pm 2.08)$. This GRI clearly separates the two classes without any overlap.

\section{Discussion}

The summary of the studies by different authors is given in Table 4. Using Heidelberg Retina Tomograph (HRT) parameters, recursive partition and regression tree and SVM classifier, Townsend et al. [54] have classified glaucoma into normal and glaucoma classes. They report a classification accuracy of $87 \%$. Fink et al. [55] used independent component analysis and K Nearest Neighbor (KNN) on HRT parameters to classify the patients into normal and glaucoma with an accuracy of $91 \%$. Multifocal -Visual Evoked Potential (M-VEP) data and Artificial Neural Network (ANN) are used to detect the glaucoma with a sensitivity of $95 \%$ and specificity of $94 \%$ [56]. Glaucoma Risk Index (GRI) is a single number which can differentiate normal fundus images from glaucoma. Bock et al. [57] have proposed GRI based on data driven approach. The pre-processed fundus images features are extracted and applied to PCA for reduction in dimensionality and classified using SVM. They report an accuracy of $80 \%$. Morphological segmentation techniques and ANN was used to identify glaucoma with $100 \%$ sensitivity and $80 \%$ specificity [32]. Huang et al. [58] used the retinal nerve fiber layer

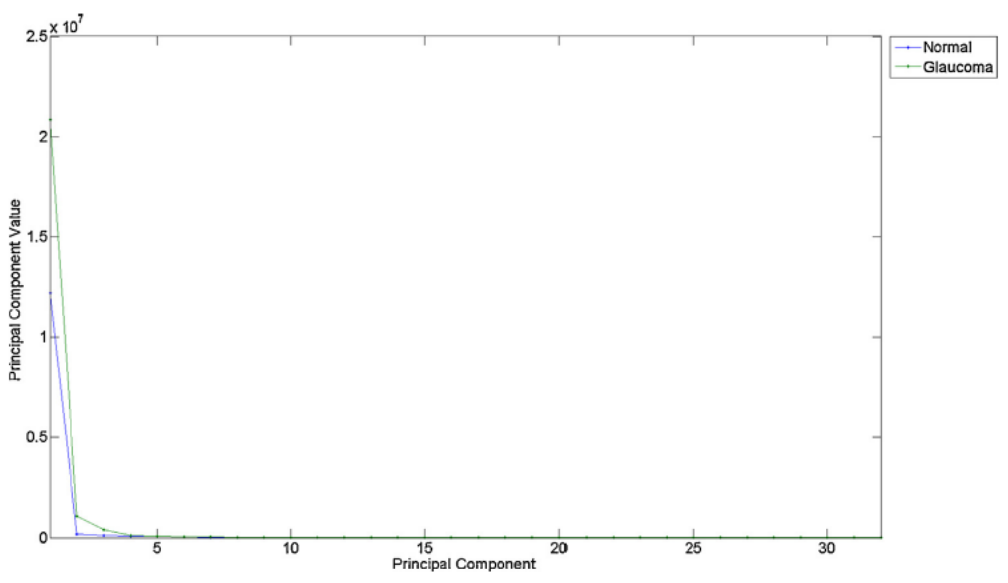

Fig. 4. Plot of variance of PCs versus number of PCs for normal and glaucoma classes.

Link to Full-Text Articles :

http://www.sciencedirect.com/science/article/pii/S1746809414001396 
www.researchgate.net/profile/Kevin Noronha2/publication/266320674 Decision support system for the glau coma using Gabor_transformation/links/543516960cf2bf1f1f27ee73.pdf 